\title{
Soluções de equações diofantinas com coeficientes nos inteiros gaussianos por meio de planilhas eletrônicas
}

\section{Solutions of diophantine equations with coefficients in Gaussian integers using electronic spreadsheets}

Laerte Bemm

Universidade Estadual de Maringá (UEM), Departamento de Matemática, Programa de Pós-Graduação em Matemática em Rede Nacional (PROFMAT), Maringá, PR, Brasil

(iD) https://orcid.org/0000-0002-0326-7662, 1bemm2@uem.br

Vinícius Bomfim Cardoso

Universidade Estadual de Maringá (UEM), Departamento de Matemática, Programa de Pós-Graduação em Matemática em Rede Nacional (PROFMAT), Maringá, PR, Brasil https : //orcid.org/0000-0002-9744-5473, viniciuscardoso92@hotmail.com

Priscila Costa Ferreira de Jesus Bemm Universidade Estadual de Maringá (UEM), Departamento de Matemática, Maringá, PR, Brasil iD https://orcid.org/0000-0003-1998-5973, pcf jbemm2@uem.br

\section{Como citar este artigo}

BEMM, Laerte; CARDOSO, Vinícius Bomfim; BEMM, Pricila Costa Ferreira de Jesus. Soluções de equações diofantinas com coeficientes nos inteiros gaussianos por meio de planilhas eletrônicas.

REMAT: Revista Eletrônica da Matemática, Bento Gonçalves, RS, v. 6, n. 2, p. e4004, 30 set. 2020. DOI: https://doi.org/10.35819/remat2020v6i2id4150

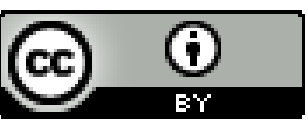

Histórico do Artigo

Submissão: 21 de abril de 2020.

Aceite: 20 de julho de 2020.

\section{Palavras-chave}

Máximo Divisor Comum

Equações Diofantinas

Inteiros Gaussianos

Domínios Euclidianos

Soluções em Planilha Eletrônicas

\section{Resumo}

Neste trabalho estudamos condições necessárias e suficientes para que uma equação diofantina linear sobre um domínio euclidiano tenha solução. Apresentamos uma série de algoritmos (funções) que podem ser implementados em planilhas eletrônicas (por exemplo LibreOffice Calc, Microsoft Excel, etc.), com o intuito de determinar (caso existam) soluções de equações diofantinas sobre $\mathbb{Z}[i]$.

\section{Keywords}

Greatest Common Divisor

Diophantine Equations

Gaussian Integers

Euclidean Domains

Spreadsheet Solution

\begin{abstract}
In this work we study necessary and sufficient conditions for a linear diophantine equation over an euclidean domain to have a solution. We present a series of algorithms (functions) that can be implemented in spreadsheets (for example LibreOffice Calc, Microsoft Excel, etc.), in order to determine (if any) solutions for Diophantine equations over $\mathbb{Z}[i]$.
\end{abstract}




\section{Introdução}

Equações lineares do tipo $a X+b Y=c, \operatorname{com} a, b, c \in \mathbb{Z}$ são comumente estudadas em disciplinas de Teoria dos Números nos cursos de graduação em Matemática. O interesse central no estudo dessas equações é encontrar soluções inteiras, ou seja, pares de números $x, y \in \mathbb{Z}$ tais que $a x+b y=c$. Essas equações são chamadas equações diofantinas em homenagem a Diophanto de Alexandria ( $\approx 250$ d. C.) que foi o primeiro a considerá-las. Quando se estuda as equações diofantinas, percebe-se que três coisas são cruciais e indispensáveis: divisibilidade, máximo divisor comum e algoritmo da divisão (em $\mathbb{Z}$ ). Como a determinação do máximo divisor comum de dois inteiros pode ser obtido por meio de sucessivas divisões (veja Hefez (2016) ou Milies e Coelho (2001)), podemos afirmar que o algoritmo da divisão é essencial para o estudo dessas equações. Portanto, é mais conveniente estudá-las onde vale um tal algoritmo. De fato, além de $\mathbb{Z}$, existem outros domínios de integridade onde vale um algoritmo da divisão. Tais domínios são chamados de euclidianos e há uma vasta literatura sobre eles (veja Anderson e Feil (2015), e Gathen e Gerhard (2013), por exemplo). Mais ainda, no Teorema 4.10 de Gathen e Gerhard (2013), os autores apresentam uma condição necessária e suficiente para que uma equação diofantina, sobre um domínio euclidiano, tenha solução. Nós apresentamos este teorema e sua prova na Proposição 4.2.

O ponto forte do estudo de equações diofantinas é que a maioria dos cálculos são algorítmicos e muitas vezes iterativos. O ponto fraco é que, em geral, tais cálculos são exaustivos e em grande quantidade. Por isso, é importante desenvolver modelos computacionais que possam ser implementados em dispositivos que resolvam tais equações. Em Santos (2016), o autor implementa, para a plataforma Android, um aplicativo de resolução de equações diofantinas com coeficientes em $\mathbb{Z}$ e em SEMATIC SCHOLAR (2019), o autor apresenta alguns algoritmos em linguagem de MATLAB para resolver equações diofantinas com coeficientes polinomiais.

Neste trabalho apresentamos uma revisão bibliográfica sobre resolução de equações diofantinas sobre domínios euclidianos e desenvolvemos uma série de algoritmos que implementamos em planilhas eletrônicas do LibreOffice para determinarmos soluções de equações diofantinas sobre o domínio do inteiros gaussianos $\mathbb{Z}[i]$. Para tanto, estruturamos o artigo da seguinte forma: na Seção 2, definimos divisibilidade, máximo divisor comum e provamos o Lema de Euclides para domínios quaisquer. Na Seção 3, apresentamos uma prova algorítmica do Teorema de Bezout para domínios 
euclidianos e algumas consequências desse que serão utilizadas na Seção 4, onde apresentamos uma condição necessária e suficiente para que uma equação diofantina tenha solução. Na mesma seção, mostramos como determinar todas as soluções. Na Seção 5, aplicamos a teoria das seções anteriores para resolver equações diofantinas com coeficientes em $\mathbb{Z}[i]$. Na Seção 6, descrevemos uma série de algoritmos que implementamos em uma planilha eletrônica do LibreOffice e que permitem obter o quociente e o resto da divisão de dois inteiros gaussianos, um máximo divisor comum de dois elementos de $\mathbb{Z}[i]$, as constantes de Bezout e as soluções de uma equação diofantina sobre $\mathbb{Z}[i]$. Finalizamos com uma breve conclusão.

Neste momento, vale ressaltar que os conceitos e resultados apresentados nas seções 2, 3 e 4 não são originais. Nós demonstramos a maioria dos resultados dessas seções por comodidade ao leitor e para deixar o trabalho o mais auto suficiente possível. Por outro lado, os algoritmos apresentados na Seção 6 são originas e acreditamos que o leitor não terá dificuldades em implementá-los nas suas atividades.

\section{Máximo divisor comum em domínios de integridade}

Esta seção é dedicada a fixarmos algumas notações, definições e resultados que são bem conhecidos e que usaremos no decorrer do trabalho. A menos que se mencione o contrário, $A$ denotará um domínio de integridade, ou seja, um anel comutativo com identidade 1 em que o produto de elementos não nulos é não nulo. Para simplificar a nomenclatura, tais anéis serão chamados de domínios. Denotaremos por $A^{*}$ ao conjunto $A-\{0\}$. Embora alguns conceitos que vamos estudar possam ser definidos para anéis em geral, interessa-nos apenas os domínios.

Exemplo 2.1. $O$ anel dos inteiros $\mathbb{Z}$ e o anel de polinômios sobre um corpo, com as operações usuais de adição e multiplicação, são domínios.

Exemplo 2.2. O conjunto $\mathbb{Z}[i]=\{a+b i: a, b \in \mathbb{Z}\}$, com as operações usuais de adição e multiplicação de números complexos, é um domínio e é chamado de domínio dos inteiros gaussianos, em homenagem a C. F. Gauss, que foi o primeiro a considerar estes números para resolver problemas de Aritmética.

Exemplo 2.3. O conjunto $\mathbb{Z}[\sqrt{\alpha}]=\{a+b \sqrt{\alpha}: a, b \in \mathbb{Z}\}$ (onde $\alpha$ é qualquer inteiro livre de quadrados) é um domínio com as operações de adição e multiplicação definidas por

$$
\begin{gathered}
(a+b \sqrt{\alpha})+(c+d \sqrt{\alpha})=(a+b)+(c+d) \sqrt{\alpha} \\
(a+b \sqrt{\alpha})(c+d \sqrt{\alpha})=(a b+\alpha b d)+(a d+b c) \sqrt{\alpha} .
\end{gathered}
$$


Para $\alpha=-1$, temos $\mathbb{Z}[\sqrt{-1}]=\mathbb{Z}[i]$. Para maiores detalhes, veja o Cap. 31 de Anderson e Feil (2015).

Definição 2.4. Sejam $A$ um domínio e $a, b \in A$. Dizemos que a divide $b$ (ou que $a$ um divisor de $b$ ou ainda $b$ é um múltiplo de a) se existe $c \in A$ tal que $b=a c$. Nesse caso, escrevemos $a \mid b$.

Observação 2.5. Note que 0 apenas divide 0 . Além disso, por A ser domínio, segue facilmente que se $a \mid b$ e $a \neq 0$, então existe um único $c \in A$ tal que $b=a c$. Tal $c$ é denotado por $\frac{b}{a}$.

A demonstração do próximo resultado será omitida por ser idêntica a da Proposição 2.1.4 de Milies e Coelho (2001).

Proposição 2.6. Sejam $a, b, c, d \in A$. Então:

(i) $a \mid a$;

(ii) Se $a \mid b$ e $b \mid c$, então $a \mid c$;

(iii) Se $a \mid b$ e $c \mid d$, então $(a c) \mid(b d)$. Em particular, se $a \mid b$, então $a c \mid b c$;

(iv) Se $a \mid b$ e $a \mid c$, então $a \mid(b s \pm c t)$ para quaisquer $s, t \in A$.

Definição 2.7. Sejam $a, b \in A$ não ambos nulos. Um elemento $d \in A$ é um máximo divisor comum $(m d c)$ de $a$ e $b$, se $d$ satisfizer as seguintes propriedades:

(i) $d \mid a$ e $d \mid b$;

(ii) Se $d^{\prime} \in A$ é tal que $d^{\prime} \mid a$ e $d^{\prime} \mid b$, então $d^{\prime} \mid d$.

Pela definição anterior, em qualquer domínio $A, m d c(a, 0)=a$, para todo $a \in A^{*}$. Também, se $b \mid a$, com $b \neq 0$, então $m d c(a, b)=b$. Por convenção, definimos $m d c(0,0)=0$. Outro ponto da definição anterior que vale destaque é que ela não garante a existência tampouco a unicidade de um $m d c$ de dois elementos. Quando existir um $m d c$ de quaisquer dois elementos, diremos que 0 domínio é com $m d c$. Por exemplo, $\mathbb{Z}$ é um domínio com $m d c$ (veja Hefez (2016) ou Milies e Coelho (2001)).

Observação 2.8. Se $d$ e $d^{\prime}$ são dois máximos divisores comuns de $a, b \in A$, então $d \mid d^{\prime}$ e $d^{\prime} \mid d$. Neste caso, existe $u \in \mathcal{U}(A)$ (conjunto dos elementos invertíveis de $A$ ) tal que $d^{\prime}=d u$. Ou seja, cada elemento da forma $d u$, com $u \in \mathcal{U}(A)$, também é um $m d c$ de a e $b$. Isto nos permite "escolher" um mdc de acordo com nossa conveniência. Em $\mathbb{Z}$, por exemplo, nós escolhemos o mdc entre a e b, como 
sendo positivo, mesmo sabendo que seu oposto também é um mdc. Para simplificar, denotamos qualquer $m d c$ de $a$ e $b$ por $m d c(a, b)$. Assim, quando escrevemos $d=m d c(a, b)$, queremos dizer que $d$ é um elemento de A que satisfaz a Definição 2.7. Quando $m d c(a, b) \in \mathcal{U}(A)$, ou equivalentemente, $m d c(a, b)=1$, dizemos que $a$ e $b$ são coprimos ou primos entre si.

Lema 2.9. (Lema de Euclides) Sejam A um domínio e $a, b \in A$, tais que $a=b q+r$ para certos $q, r \in A$. Se $m d c(b, r)$ existe, então $m d c(a, b)$ existe e $m d c(a, b)=m d c(b, r)$.

Demonstração. Se existe $d=m d c(b, r)$, então existem $m, n \in A$ tal que $b=d m$ e $r=d n$. Assim, $a=(d m) q+d n=d(m q+n)$, isto é, $d \mid a$. Agora, se $d^{\prime} \in A$ é tal que $d^{\prime} \mid a$ e $d^{\prime} \mid b$, então existem $a^{\prime}, b^{\prime} \in A$ tais que, $a=d^{\prime} a^{\prime}$ e $b=d^{\prime} b^{\prime}$. Daí, $r=a-b q=d^{\prime}\left(a^{\prime}-b^{\prime} q\right)$, o que implica, $d^{\prime} \mid r$. Como $d=m d c(b, r)$, temos da Definição 2.7 que $d^{\prime} \mid d$. Logo, $m d c(a, b)$ existe e $m d c(a, b)=d=m d c(b, r)$.

\section{Domínios euclidianos}

Para os propósitos deste artigo, uma importante classe de domínios são os domínios euclidianos, isto é, aqueles que admitem um algoritmo da divisão.

Definição 3.1. Seja A um domínio. Diremos que A é um domínio euclidiano, se existe uma função $\delta: A^{*} \rightarrow \mathbb{N}$ que satisfaça as seguintes propriedades:

(i) $\forall a, b \in A^{*}$, se $b \mid a$, então $\delta(b) \leq \delta(a)$;

(ii) $\forall a, b \in A$ com $b \neq 0$, existem $q, r \in A$ tais que $a=b q+r$, com $r=0$ ou $\delta(r)<\delta(b)$.

Nomenclatura. No item (ii) da definição anterior, a é o dividendo, $b$ é o divisor, q o quociente e $r$ o resto. A função $\delta$ é denominada função norma.

Exemplo 3.2. $\mathbb{Z}$ é um domínio euclidiano com a função norma $\delta: \mathbb{Z}^{*} \rightarrow \mathbb{N}$ dada por $\delta(a)=|a|$.

Exemplo 3.3. Se $\mathbb{K}$ é um corpo, então o anel de polinômios $\mathbb{K}[x]$ é um domínio euclidiano com a função norma $\delta: \mathbb{K}[x]^{*} \rightarrow \mathbb{N}$ dada por $\delta(p(x))=\operatorname{gr}(p(x))$ (grau de $p(x)$ ).

Exemplo 3.4. O domínio dos inteiros gaussianos $\mathbb{Z}[i]$ é euclidiano com a função norma $\delta: \mathbb{Z}[i]^{*} \rightarrow \mathbb{N}$ definida por $\delta(a+b i)=a^{2}+b^{2}$. De fato, note que para quaisquer $z, w \in \mathbb{Z}[i], \delta(z w)=\delta(z) \delta(w)$. Assim, se $z_{1}, z_{2} \in \mathbb{Z}[i]^{*}$ são tais que $z_{1} \mid z_{2}$, então $z_{2}=z_{1} z_{3}$ e daí $\delta\left(z_{2}\right)=\delta\left(z_{1}\right) \delta\left(z_{3}\right) \geq \delta\left(z_{1}\right)$. Isto mostra o item $(i)$ da Definição 3.1. Para mostrar (ii), sejam $z_{1}, z_{2} \in \mathbb{Z}[i]$ com $z_{2} \neq 0$. Vamos mostrar que existem $q, r \in \mathbb{Z}[i]$ tais que $z_{1}=q z_{2}+r \operatorname{com} r=0$ ou $\delta(r)<\delta\left(z_{2}\right)$. Com efeito, como $z_{1}, z_{2} \in \mathbb{C}$, 
existem $\alpha, \beta \in \mathbb{Q}$ tais que $\frac{z_{1}}{z_{2}}=\alpha+\beta i$. Considere $m, n \in \mathbb{Z}$ tais que $|m-\alpha| \leq 0,5$ e $|n-\beta| \leq 0,5$. Então, $z_{1}=z_{2}(\alpha+\beta i)=z_{2}(\alpha-m+m+\beta i-n i+n i)=z_{2}(m+n i)+z_{2}[(\alpha-m)+(\beta-n) i]$. Tomando $q=m+n i$ e $r=z_{2}[(\alpha-m)+(\beta-n) i]$, temos $z_{1}=z_{2} q+r$. Mais ainda, como $m, n \in \mathbb{Z}$, segue que $q=m+n i \in \mathbb{Z}[i]$ e por consequência, $r=z_{1}-z_{2} q \in \mathbb{Z}[i]$. Finalmente,

$$
\delta(r)=\delta\left(z_{2}\right)\left[(\alpha-m)^{2}+(\beta-n)^{2}\right] \leq \delta\left(z_{2}\right)(0,25+0,25)<\delta\left(z_{2}\right)
$$

A seguir apresentamos o algoritmo da divisão de $\mathbb{Z}[i]$ de maneira sucinta.

Algoritmo 3.5. Sejam $z_{1}=x+y i, z_{2}=a+b i \in \mathbb{Z}[i]$, com $z_{2} \neq 0$.

Passo 1. Calcular $\frac{z_{1}}{z_{2}}=\frac{x a+y b}{a^{2}+b^{2}}+\frac{(y a-x b)}{a^{2}+b^{2}} i$ e escrever $\alpha:=\frac{x a+y b}{a^{2}+b^{2}}$ e $\beta:=\frac{y a-x b}{a^{2}+b^{2}} ;$

Passo 2. Tome $m, n \in \mathbb{Z}$ tais que $|m-\alpha| \leq 0,5$ e $|n-\beta| \leq 0,5$.

Resultado: O quociente é $q=m+n i$ e o resto é $r=z_{2}[(\alpha-m)+(\beta-n) i]$.

Observação 3.6. Se $A$ é um domínio euclidiano com norma $\delta$, então para todo $a \in A^{*}, \delta(a) \geq 1 e$ $\mathcal{U}(A)=\left\{a \in A^{*}: \delta(a)=\delta(1)\right\}$. Assim, por exemplo, $\mathcal{U}(\mathbb{Z}[i])=\{1,-1, i,-i\}$ e $\mathcal{U}(\mathbb{K}[x])=\mathbb{K}^{*}$. Em particular, disso e da Observação 2.8 , temos que se $a, b \in \mathbb{Z}[i]$ e $d=m d c(a, b)$, então $-d$, di e - di também são $m d c(a, b)$.

Observação 3.7. Note que todo domínio euclidiano é um domímio com mdc. De fato, sejam A um domínio euclidiano com norma $\delta$ e $a, b \in A$.

Definimos $r_{0}:=a$ e $r_{1}:=b$. Existem $q_{1}, r_{2} \in A$ tais que $r_{0}=r_{1} q_{1}+r_{2}$, com $r_{2}=0$ ou $\delta\left(r_{2}\right)<\delta\left(r_{1}\right)$.

Se $r_{2}=0$, então $r_{1} \mid r_{0}$ e temos $\operatorname{mdc}(a, b)=\operatorname{mcd}\left(r_{0}, r_{1}\right)=r_{1}$.

Se $r_{2} \neq 0$, existem $q_{2}, r_{3} \in A$ tais que $r_{1}=r_{2} q_{2}+r_{3}$, com $r_{3}=0$ ou $\delta\left(r_{3}\right)<\delta\left(r_{2}\right)$.

Se $r_{3}=0$, então $r_{2} \mid r_{1}$ e temos $m d c(a, b)=\operatorname{mcd}\left(r_{0}, r_{1}\right)=m d c\left(r_{1}, r_{2}\right)=r_{2}$.

Se $r_{3} \neq 0$, repetimos o processo.

Como $\delta\left(r_{1}\right)>\delta\left(r_{2}\right)>\delta\left(r_{3}\right)>\cdots$ é uma sequência decrescente de números naturais, em algum momento obteremos um resto $r_{n}=0$. Nesse caso, teremos $r_{n-2}=r_{n-1} q_{n-1}$, ou seja, $r_{n-1} \mid r_{n-2}$. Então, $m d c(a, b)=m d c\left(r_{0}, r_{1}\right)=m d c\left(r_{1}, r_{2}\right)=m d c\left(r_{2}, r_{3}\right)=\cdots=m d c\left(r_{n-2}, r_{n-1}\right)=r_{n-1}$.

A tabela a seguir representa um esquema prático para o cálculo de um $m d c(a, b)$. No momento em que numa das sucessivas divisões tivermos um resto $r_{n}=0$, teremos $r_{n-1}=m d c(a, b)$.

Exemplo 3.8. Pelas figuras a seguir, temos $m d c(-72+56 i, 25-33 i)=1-i$ e $m d c(1250+885 i, 720+256 i)=-1$, ou seja, $1250+885 i$ e $720+256 i$ são coprimos. 
Tabela 1 - Esquema prático para o cálculo do $m d c(a, b)$.

\begin{tabular}{|c|c|c|c|c|c|c|c|c|c|}
\hline$k$ & 0 & 1 & 2 & 3 & 4 & $\cdots$ & $n-2$ & $n-1$ & $n$ \\
\hline$r_{k}$ & $r_{0}=a$ & $r_{1}=b$ & $r_{2}$ & $r_{3}$ & $r_{4}$ & $\cdots$ & $r_{n-2}$ & $r_{n-1}$ & $r_{n}=0$ \\
\hline$q_{k}$ & - & $q_{1}$ & $q_{2}$ & $q_{3}$ & $q_{4}$ & $\cdots$ & $q_{n-2}$ & $q_{n-1}$ & - \\
\hline
\end{tabular}

Figura $1-m d c(-72+56 i, 25-33 i)$.

\begin{tabular}{c|c|c|c|c|c|c|c|}
\hline$k$ & 0 & 1 & 2 & 3 & 4 & 5 & 6 \\
\hline$r_{k}$ & $-72+56 i$ & $25-33 i$ & $11+15 i$ & $6+4 i$ & $3+i$ & $1-i$ & 0 \\
\hline $\mathrm{q}_{k}$ & & $-2-i$ & $-1-2 i$ & $2+i$ & $2+i$ & $1+2 i$ & Acabou \\
\hline
\end{tabular}

Fonte: Elaboração dos autores (2020).

Teorema 3.9. (Teorema de Bezout) Sejam $A$ um domínio euclidiano e $a, b \in A$. Então, existem $s, t \in A$ (chamadas de constantes de Bezout) tais que $a s+b t=m d c(a, b)$.

Demonstração. A demonstração explicita $s$ e $t$ de maneira algorítmica e recursiva. Consideremos as notações (para restos e quocientes) e os cálculos feitos na Observação 3.7. Claramente, temos $r_{k}=r_{k-2}-r_{k-1} q_{k-1}$, para todo $k \geq 2$. Consideremos também as seguintes fórmulas de recorrência:

$$
\begin{gathered}
s_{0}=1 \text { e } t_{0}=0 ; \\
s_{1}=0 \text { e } t_{1}=1 ; \\
s_{k}=s_{k-2}+s_{k-1}\left(-q_{k-1}\right), \quad \forall k \geq 2 \\
\mathrm{e} \\
t_{k}=t_{k-2}+t_{k-1}\left(-q_{k-1}\right), \quad \forall k \geq 2 .
\end{gathered}
$$

Afirmação: Para todo $k \geq 2, r_{k}=a s_{k}+b t_{k}$.

De fato, para $k=2, r_{2}=a-b q_{1}=a+b\left(-q_{1}\right)=a s_{2}+b t_{2}$. Se supormos que $r_{j}=a s_{j}+b t_{j}$, para todo $2 \leq j \leq k-1$ (hipótese de indução), então

$$
r_{k}=r_{k-2}-q_{k-1} r_{k-1}=\left(a s_{k-2}+b t_{k-2}\right)-q_{k-1}\left(a s_{k-1}+b t_{k-1}\right)=a s_{k}+b t_{k} .
$$

Disso segue que:

1. Se $r_{2}=0$, então $\operatorname{mcd}(a, b)=b=0 a+1 b$. Nesse caso, $s=0$ e $t=1$, i. é, $s=s_{1}$ e $t=t_{1}$.

2. Se $r_{2} \neq 0$ e $r_{3}=0$, então $m d c(a, b)=r_{2}=a s_{2}+b t_{2}$. Nesse caso, $s=s_{2}$ e $t=t_{2}$.

3. Se $r_{3} \neq 0$ e $r_{4}=0$ então $m d c(a, b)=r_{3}=a s_{3}+b t_{3}$. Nesse caso, $s=s_{3}$ e $t=t_{3}$.

4. Se necessário, esse processo pode ser repetido e paramos quando algum $r_{n}=0$. Então, obtemos $m d c(a, b)=r_{n-1}=a s+b t, \operatorname{com} s=s_{n-1}=s_{n-2}\left(-q_{n-2}\right)+s_{n-3}$ e $t=t_{n-1}=t_{n-2}\left(-q_{n-2}\right)+t_{n-3}$. 
Figura $2-m d c(1250+885 i, 720+256 i)$.

\begin{tabular}{c|c|c|c|c|c|c|c|c|c|c|c|}
\hline $\mathrm{k}$ & 0 & 1 & 2 & 3 & 4 & 5 & 6 & 7 & 8 & 9 & 10 \\
\hline $\mathrm{r}_{\mathrm{k}}$ & $1250+885 \mathrm{i}$ & $720+256 \mathrm{i}$ & $66-347 \mathrm{i}$ & $26+124 \mathrm{i}$ & $20+51 \mathrm{i}$ & $-14+22 \mathrm{i}$ & $-10+\mathrm{i}$ & 4 & $-2+\mathrm{i}$ & -1 & 0 \\
\hline $\mathrm{q}_{\mathrm{k}}$ & & $2+\mathrm{i}$ & $2 \mathrm{i}$ & $-3-\mathrm{i}$ & 2 & $1-2 \mathrm{i}$ & $2-2 \mathrm{i}$ & -2 & $-2-\mathrm{i}$ & $2-\mathrm{i}$ & Acabou \\
\hline
\end{tabular}

Fonte: Elaboração dos autores (2020).

Observação 3.10. A demonstração anterior pode ser melhor visualizada em uma tabela como a seguinte.

Tabela 2 - Representação da demonstração do Teorema de Bezout.

\begin{tabular}{|c|c|c|c|l|c|l|c|c|}
\hline$k$ & 0 & 1 & 2 & $\cdots$ & $k$ & $\cdots$ & $n-1$ & $n$ \\
\hline$r_{k}$ & $r_{0}=a$ & $r_{1}=b$ & $r_{2}$ & $\cdots$ & $r_{k}$ & $\cdots$ & $r_{n-1}$ & $r_{n}=0$ \\
\hline$q_{k}$ & - & $q_{1}$ & $q_{2}$ & $\cdots$ & $q_{k}$ & $\cdots$ & $q_{n-1}$ & - \\
\hline$s_{k}$ & 1 & 0 & 1 & $\cdots$ & $s_{k-1}\left(-q_{k-1}\right)+s_{k-2}$ & $\cdots$ & $s_{n-2}\left(-q_{n-2}\right)+s_{n-3}$ & - \\
\hline$q_{k}$ & 0 & 1 & $-q_{1}$ & $\cdots$ & $t_{k-1}\left(-q_{k-1}\right)+t_{k-2}$ & $\cdots$ & $t_{n-2}\left(-q_{n-2}\right)+t_{n-3}$ & - \\
\hline
\end{tabular}

Exemplo 3.11. Em $\mathbb{Z}[i]$, consideremos $a=132+88 i$ e $b=42+18 i$. Pela figura a seguir, temos que $m d c(132+88 i, 42+18 i)=2-2 i$ e as constantes de Bezout são $s=4+i$ e $t=-13-6 i$.

Figura $3-m d c$ e constantes de Bezout para $132+88 i$ e $42+18 i$.

\begin{tabular}{c|c|c|c|c|c|c|}
\hline$k$ & 0 & 1 & 2 & 3 & 4 & 5 \\
\hline$r_{k}$ & $132+88 i$ & $42+18 i$ & $24-8 i$ & $10+2 i$ & $2-2 i$ & 0 \\
\hline$q_{k}$ & & $3+i$ & $1+i$ & $2-i$ & $2+3 i$ & Acabou \\
\hline$s_{k}$ & 1 & 0 & 1 & $-1-i$ & $4+i$ & Acabou \\
\hline$t_{k}$ & 0 & 1 & $-3-i$ & $3+4 i$ & $-13-6 i$ & Acabou \\
\hline
\end{tabular}

Fonte: Elaboração dos autores (2020).

Exemplo 3.12. Observe na figura a seguir que o mdc de dois inteiros gaussianos pode ser um número inteiro e as constantes de Bezout não serem números inteiros. 
Figura $4-m d c$ e constantes de bezout para $132+88 i$ e $52+32 i$.

\begin{tabular}{c|c|c|c|c|c|c|c|}
\hline$k$ & 0 & 1 & 2 & 3 & 4 & 5 & 6 \\
\hline$r_{k}$ & $132+88 i$ & $52+32 i$ & $-24-8 i$ & $12-8 i$ & $-4-4 i$ & 4 & 0 \\
\hline$q_{k}$ & & 3 & $-2-i$ & $-1-i$ & $2 i$ & $-1-i$ & Acabou \\
\hline$s_{k}$ & 1 & 0 & 1 & $2+i$ & $2+3 i$ & $8-3 i$ & Acabou \\
\hline$t_{k}$ & 0 & 1 & -3 & $-5-3 i$ & $-5-8 i$ & $-21+7 i$ & Acabou \\
\hline
\end{tabular}

Fonte: Elaboração dos autores (2020).

Temos que $m d c(132+88 i, 52+32 i)=4$ e as constantes de Bezout são $s=8-3 i$ e $t=-21+7 i$.

Corolário 3.13. Sejam A um domínio euclidiano e $a, b, c \in A$. Então $m d c(c a, c b)=c \cdot m c d(a, b)$.

Demonstração. Seja $d=m d c(a, b)$. Como $d \mid a$ e $d \mid b$, segue da Proposição 2.6(iii) que $c d \mid a c$ e $c d \mid b c$. Mais ainda, pelo Teorema de Bezout, existem $s, t \in A$ tais que $d=a s+b t$. Daí, $c d=c a s+c b t$. Agora, se $d^{\prime} \in A$ é tal que $d^{\prime} \mid a c$ e $d^{\prime} \mid b c$, então pela Proposição 2.6(iv), $d^{\prime} \mid a c s+b c s=c d$.

Observação 3.14. Sejam $A$ um domínio euclidiano e $a, b \in A$ tais que $d=m d c(a, b) \neq 0$. Então $a=d \frac{a}{d}$ e $b=d \frac{b}{d}$ e pelo corolário anterior, $d=m d c(a, b)=m d c\left(d \frac{a}{d}, d \frac{b}{d}\right)=d \cdot m d c\left(\frac{a}{d}, \frac{b}{d}\right)$. Como $A$ é domínio e $d \neq 0$, temos $m d c\left(\frac{a}{d}, \frac{b}{d}\right)=1$.

Corolário 3.15. Sejam A um domínio euclidiano e $a, b, c \in A$ tais que $m d c(a, b)=1$ e $a \mid b c$, então $a \mid c$.

Demonstração. De fato, se $m d c(a, b)=1$, então pelo Corolário $3.13 m d c(a c, b c)=c$. Como $a \mid a c$ e $a \mid b c$, temos da Definição 2.7 $(i i)$ que $a \mid c$.

\section{Equações diofantinas em domínios euclidianos}

Sejam $A$ um domínio euclidiano e $a, b \in A$. Pelo que vimos na seção anterior, existem $s, t \in A$ tais que $a s+b t=d$. Isso significa que a equação linear $a X+b Y=m d c(a, b)$ tem solução em $A$. De modo mais geral, temos:

Definição 4.1. Sejam $A$ um domínio e $a, b, c \in A$ elementos não nulos. Uma equação do tipo

$$
a X+b Y=c
$$

é chamada de Equação Diofantina Linear sobre $A$ nas variáveis $X$ e $Y$. Os elementos $a$ e $b$ são chamados coeficientes e $c$ de termo independente. Se existem $x, y \in A$ tais que $a x+b y=c$, dizemos que (1) tem solução (em A). 
Em cursos de Teoria dos Números, é comum se estudar equações diofantinas do tipo (1), $\operatorname{com} a, b, c \in \mathbb{Z}$. Nosso objetivo nesta seção é estudar essas equações sobre um domínio euclidiano qualquer e aplicar esta teoria a equações com coeficientes em $\mathbb{Z}[i]$.

Proposição 4.2. Sejam $A$ um domínio euclidiano, $a, b, c \in A$ e $d=m d c(a, b) \neq 0$. Uma equação diofantina $a X+b Y=c$ tem solução em $A$ se e somente se $d \mid c$. Em particular, se $a$ e $b$ são coprimos, então para todo $c \in A, a X+b Y=c$ tem solução em $A$.

Demonstração. Suponhamos que $a X+b Y=c$ tenha solução em $A$. Então, existirão $x, y \in A$ tais que $a x+b y=c$. Como $d \mid a$ e $d \mid b$, segue da Proposição 2.6(iv) que $d \mid c$. Reciprocamente, se $d \mid c$ então, $c=d \frac{c}{d}$. Pelo Teorema de Bezout, existem $s, t \in A$, tais que $a s+b t=d$. Multiplicando ambos os lados por $\frac{c}{d}$, obtemos $a\left(\frac{c}{d} s\right)+b\left(\frac{c}{d} t\right)=d \frac{c}{d}=c$, e a equação $a X+b Y=c$ tem solução em $A$.

Note que podemos encontrar algoritmicamente uma solução de $a X+b Y=c$, quando $d \mid c$. De fato, através de divisões sucessivas, podemos encontrar $s, t \in A$ tais que $a s+b t=d$. Então, $x_{0}=s \frac{c}{d}, y_{0}=t \frac{c}{d}$ é uma solução em $A$, chamada uma solução inicial.

Teorema 4.3. Sejam $A$ um domínio euclidiano, $d=m d c(a, b) \neq 0$ e $a X+b Y=c$ uma equação diofantina em $A$ tal que $d \mid c$. Escrevendo $d=a s+b t$, com $s, t \in A$, temos:

1. $x_{0}=\frac{c}{d} s, y_{0}=\frac{c}{d} t$ é solução $a X+b Y=c$

2. Para todo $z \in A, x=x_{0}+\frac{b}{d} z, y=y_{0}-\frac{a}{d} z$ é solução de $a X+b Y=c$;

3. Se $x^{\prime}, y^{\prime}$ é outra solução de $a X+b Y=c$, então existe $z \in A$ tal que $x^{\prime}=x_{0}+\frac{b}{d} z, y^{\prime}=y_{0}-\frac{a}{d} z$.

\section{Demonstração. 1. Segue da Proposição 4.2 .}

2. Substituindo $x=x_{0}+\frac{b}{d} z, y=y_{0}-\frac{a}{d} z$ na equação $a X+b Y=c$, verificamos que é solução.

3. Sejam $x^{\prime}, y^{\prime} \in A$ uma solução de $a X+b Y=c$. Escrevemos $a_{1}=\frac{a}{d}$ e $b_{1}=\frac{b}{d}$. Então

$$
\begin{aligned}
a x^{\prime}+b y^{\prime}=a x_{0}+b y_{0} & \Rightarrow a\left(x^{\prime}-x_{0}\right)=b\left(y_{0}-y^{\prime}\right) \\
& \Rightarrow d a_{1}\left(x^{\prime}-x_{0}\right)=d b_{1}\left(y_{0}-y^{\prime}\right) \\
& \Rightarrow a_{1}\left(x^{\prime}-x_{0}\right)=b_{1}\left(y_{0}-y^{\prime}\right), \text { pois } A \text { é domínio } \\
& \Rightarrow b_{1} \mid a_{1}\left(x^{\prime}-x_{0}\right) \\
& \Rightarrow b_{1} \mid\left(x^{\prime}-x_{0}\right), \text { pelo Corolário } 3.15 \\
& \Rightarrow x^{\prime}-x_{0}=b_{1} z, \text { para algum } z \in A \\
& \Rightarrow x^{\prime}=x_{0}+b_{1} z, \text { para algum } z \in A .
\end{aligned}
$$


Substituindo $x^{\prime}-x_{0}$ por $b_{1} z$ em $a_{1}\left(x^{\prime}-x_{0}\right)=b_{1}\left(y_{0}-y^{\prime}\right)$ obtemos $a_{1} b_{1} z=b_{1}\left(y_{0}-y^{\prime}\right)$, donde $y^{\prime}=y_{0}-a_{1} z$, pois $A$ é domínio. Logo, $x^{\prime}=x_{0}+\frac{b}{d} z$ e $y^{\prime}=y_{0}-\frac{a}{d} z$.

\section{Aplicação: equações diofantinas sobre $\mathbb{Z}[i]$}

Nesta seção, vamos aplicar a teoria que vimos anteriormente para estudar equações diofantinas sobre $\mathbb{Z}[i]$. Vejamos dois exemplos.

Exemplo 5.1. Considere a equação $(52+32 i) X+(15+25 i) Y=7+i$. Nesse caso, $a=r_{0}=52+32 i$, $b=r_{1}=15+25 i$ e $c=7+i$. Observando a figura a seguir, vemos que $m d c(52+32 i, 15+25 i)=-1-i$ e as constantes de Bezout são $s=s_{4}=-8+5 i$ e $t=t_{4}=10-17 i$.

Figura $5-m d c$ e constantes de Bezout para $52+32 i$ e $15+25 i$.

\begin{tabular}{c|c|c|c|c|c|c|}
\hline$k$ & 0 & 1 & 2 & 3 & 4 & 5 \\
\hline$r_{k}$ & $52+32 i$ & $15+25 i$ & $-3-3 i$ & $-2 i$ & $-1-i$ & 0 \\
\hline$q_{k}$ & & $2-i$ & $-7-2 i$ & $1-i$ & $1+i$ & Acabou \\
\hline$s_{k}$ & 1 & 0 & 1 & $7+2 i$ & $-8+5 i$ & Acabou \\
\hline$t_{k}$ & 0 & 1 & $-2+i$ & $-15+3 i$ & $10-17 i$ & Acabou \\
\hline
\end{tabular}

Fonte: Elaboração dos autores (2020).

Agora, dividindo $c=7+i$ por $-1-i=m d c(52+32 i, 25+15 i)$ obtemos o quociente $q=-4+3 i$ e resto $r=0$, ou seja, $-1-i \mid 7+i$. Logo, a equação diofantina dada tem solução e uma delas é $x_{0}=\frac{c}{d} s=(-4+3 i)(-8+5 i)=17-44 i$ e $y_{0}=\frac{c}{d} t=(-4+3 i)(10-17 i)=11+98 i$. As demais soluções são da forma $x_{z}=x_{0}+\frac{b}{d} z=(17-44 i)+(-42+10 i) z$ e $y_{z}=y_{0}-\frac{a}{d} z=(11+98 i)+(20+5 i) z$.

Exemplo 5.2. Vamos estudar a equação $(15+25 i) X+(10+18 i) Y=5-5 i$. A tabela da figura a seguir nos dá $m d c(15+25 i, 10+18 i)=1-i$ e constantes de Bezout são $s=s_{4}=5-2 i$ e $t=t_{4}=-7+3 i$.

Figura $6-m d c$ e constantes de Bezout para $15+25 i$ e $10+18 i$.

\begin{tabular}{c|c|c|c|c|c|c|}
\hline$k$ & 0 & 1 & 2 & 3 & 4 & 5 \\
\hline$r_{k}$ & $15+25 i$ & $10+18 i$ & $5+7 i$ & $4 i$ & $1-i$ & 0 \\
\hline$q_{k}$ & & 1 & 2 & $2-i$ & $-2+2 i$ & Acabou \\
\hline$s_{k}$ & 1 & 0 & 1 & -2 & $5-2 i$ & Acabou \\
\hline$t_{k}$ & 0 & 1 & -1 & 3 & $-7+3 i$ & Acabou \\
\hline
\end{tabular}

Fonte: Elaboração dos autores (2020). 
Como $1-i \mid 5-5 i$, a equação dada tem solução e uma delas é $x_{0}=5(5-2 i)=25-10 i$ e $y_{0}=5(-7+3 i)=-35+15 i$. As demais soluções são da forma $x_{z}=(25-10 i)+(-4+14 i) z e$ $y_{z}=(-35+15 i)+(5-20 i) z$. Para $z=1+i$, obtemos $x_{z}=7$ e $y_{z}=-10$. Logo, a equação $(15+25 i) X+(10+18 i) Y=5-5 i$ tem uma solução inteira.

\section{Cálculos em $\mathbb{Z}[i]$ por meio de planilhas eletrônicas}

O algoritmo da divisão para inteiros gaussianos envolve diversos cálculos que podem nos levar a cometer erros e, por consequência, não conseguirmos determinar o quociente e o resto da divisão corretamente. Como toda a teoria de equações diofantinas sobre domínios euclidianos depende basicamente do algoritmo da divisão, é de fundamental importância que tenhamos uma ferramenta que nos auxilie com os cálculos para a resolução desse tipo de equações. Durante o desenvolvimento deste trabalho, as planilhas eletrônicas se mostraram bastante satisfatórias nesse sentido. Isso se dá porque elas têm várias funções matemáticas que podem ser usadas para cálculos algébricos com números complexos, matrizes, etc. Em LIBREOFFICE (2020), encontra-se uma lista com várias funções pré-definidas do programa LibreOffice. Também há uma descrição e a sintaxe de cada função. Para acessar tais descrições, basta clicar no nome da função.

Para nós, a função "COMPLEXO" é uma das mais importantes. Ela converte um par ordenado de números reais em um número complexo. Sua sintaxe é: "COMPLEXO(NúmeroReal; INúm; Sufixo)", em que a entrada "NúmeroReal" indica a parte real do número complexo e "INúm" indica a parte imaginária. A entrada "sufixo" pode ser deixada em branco. Portanto, se em uma célula de uma planilha do LibreOffice escrevermos "=COMPLEXO(3;5)", o retorno será o número complexo $3+5 i$.

Nesta seção apresentamos uma gama de algoritmos que utilizam diversas funções prédefinidas do LibreOffice para criar novas funções. Como alguns destes algoritmos têm descrição muito longa (veja o Passo 4 do Algoritmo6.1), o leitor poderá copiá-las da versão em PDF deste trabalho e colar em uma planilha eletrônica. Para tanto, faz-se necessário seguir algumas recomendações. Primeiro, o leitor deve copiar linha por linha de cada passo dos algoritmos e colar numa planilha eletrônica. Segundo, em alguns algoritmos (veja Passo 4 do Algoritmo 6.1 aparecerá a palavra "IMAGINÁRIO". Ao copiar e colar tal palavra, o resultado será "IMAGIN'ARIO”, ou seja, o acento agudo do "A" não se mantém, igualmente no LibreOffice como está no PDF. Isso tem que ser corrigido manualmente pelo leitor na planilha. Terceiro, outro elemento que não se mantém, como no 
PDF, são as palavras com aspas. No Algoritmo 6.1, por exemplo, aparece “Acabou". Nesse caso, ao copiar e colar do PDF para o LibreOffice, as aspas não serão manidas e, consequentemente, o LibreOffice não as reconhecerá. É preciso que o leitor apague as aspas copiadas e digite-as na planilha. Por último, é necessário garantir que não haja espaços nas fórmulas das planilhas LibreOffice.

\subsection{Divisão em $\mathbb{Z}[i]$}

Para determinar o quociente e o resto da divisão de dois elementos de $\mathbb{Z}[i]$ via uma planilha eletrônica, procedemos de acordo com o seguinte algoritmo:

\section{Algoritmo 6.1.}

Passo 1: Escolha $a=a_{1}+a_{2} i$ e $b=b_{1}+b_{2} i$ em $\mathbb{Z}[i]$, com $b \neq 0$ e abra uma planilha em branco.

Passo 2: Na célula B1, digite: $=\operatorname{COMPLEXO}\left(a_{1} ; a_{2}\right)$

Passo 3: Na célula C1, digite: $=C O M P L E X O\left(b_{1} ; b_{2}\right)$

Passo 4: Na célula C2, digite:

$$
\begin{aligned}
& \text { =SE(C1=COMPLEXO(0;0);"Acabou";COMPLEXO(SE(ABS(ARREDONDAR.PARA.BAIXO(IMREAL } \\
& \text { (IMDIV(B1;C1));0)-IMREAL(IMDIV(B1;C1)))>0,5;ARREDONDAR.PARA.CIMA(IMREAL } \\
& \text { (IMDIV(B1;C1));0);ARREDONDAR.PARA.BAIXO(IMREAL(IMDIV(B1;C1));0));SE } \\
& \text { (ABS(ARREDONDAR.PARA.BAIXO(IMAGINÁRIO(IMDIV(B1;C1));0)- } \\
& \text { IMAGINÁRIO(IMDIV(B1;C1)))>0,5;ARREDONDAR.PARA.CIMA(IMAGINÁRIO } \\
& \text { (IMDIV(B1;C1));0);ARREDONDAR.PARA.BAIXO(IMAGINÁRIO(IMDIV(B1;C1));0)))) }
\end{aligned}
$$

Passo 5: Na célula D1, digite: =IMSUBTR(B1;IMPROD(C2;C1))

Resultado: As células C2 e D1 resultam, respectivamente, o quociente e o resto da divisão de a por $b$.

A figura a seguir ilustra o algoritmo anterior aplicado numa planilha eletrônica para $a=75+41 i$ e $b=9+15 i$. Obtemos o quociente $q=4-2 i$ e o resto é $r=9-i$.

Figura 7 - Quociente e resto da divisão de $75+41 i$ por $9+15 i$.

\begin{tabular}{|c|c|c|c|c|c|}
\hline 4 & A & B & C & D & E \\
\hline 1 & & $75+41 i$ & $9+15 i$ & $9-i$ & \\
\hline 2 & & & $4-2 i$ & & \\
\hline 3 & & & & & \\
\hline
\end{tabular}

Fonte: Elaboração dos autores (2020). 
A figura a seguir ilustra o contido na célula C2.

Figura 8 - Função da célula C2.

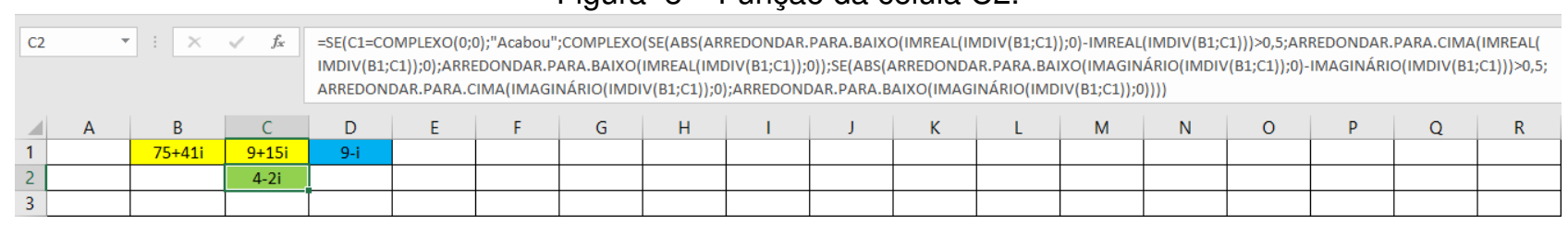

Fonte: Elaboração dos autores (2020).

\subsection{Cálculo do $m d c$ em $\mathbb{Z}[i]$}

Pelo Teorema de Bezout, para determinarmos o $m d c$ entre dois elementos de um domínio euclidiano, podemos aplicar o algoritmo da divisão sucessivas vezes até encontrarmos um resto igual a 0 . O resto anterior será o $m d c$ procurado. Portanto, podemos usar uma tabela do LibreOffice para determinar o $m d c$ de quaisquer dois inteiros gaussianos $a$ e $b$, aplicando o seguinte algoritmo:

\section{Algoritmo 6.2.}

Passo 1: Escolha $a=a_{1}+a_{2} i, b=b_{1}+b_{2} i \in \mathbb{Z}[i]$ com $b \neq 0$ e aplique o Algoritmo6.1.

Passo 2: Arraste a célula C2 sobre as células D2, E2, F2, G2, etc.

Passo 3: Arraste a célula D1 sobre as células E1, F1, G1, H1, etc.

Resultado: Quando alguma célula da linha 1 resultar 0 , o mdc de a e b será o elemento da linha 1 imediatamente anterior a essa.

A figura a seguir mostra os cálculos que determinam $m d c(75+41 i, 9+15 i)$ utilizando o algoritmo anterior. As células em azul são os sucessivos restos, enquanto que as células em verde são os respectivos quocientes. Como a célula $\mathrm{G} 1$ retornou 0 , temos o retorno da célula $\mathrm{F} 1$ é o $m d c(75+41 i, 9+15 i)$, ou seja, $m d c(75+41 i, 9+15 i)=1-i$.

Figura 9 -Cálculo de $m d c(75+41 i, 9+15 i)$.

\begin{tabular}{|c|c|c|c|c|c|c|c|c|}
\hline 4 & $A$ & $B$ & $C$ & $D$ & $E$ & $F$ & $G$ & $H$ \\
\hline 1 & & $75+41 i$ & $9+15 i$ & $9-i$ & $-2-2 i$ & $1-i$ & 0 & \\
\hline 2 & & & $4-2 i$ & $1+2 i$ & $-2+2 i$ & $-2 i$ & Acabou & \\
\hline 3 & & & & & & & & \\
\hline
\end{tabular}

Fonte: Elaboração dos autores (2020). 


\subsection{Constantes de Bezout em $\mathbb{Z}[i]$}

O algoritmo a seguir nos mostra como é possível determinar, por meio de uma tabela do LibreOffice, as constantes Bezout para um par $a, b \in \mathbb{Z}[i]$, ou seja, $s, t \in \mathbb{Z}[i]$ tais que $a s+b t=$ $m d c(a, b)$. Para isso, utilizamos as fórmulas recursivas dadas na demonstração do Teorema de Bezout (ver Passos 4 e 5).

\section{Algoritmo 6.3.}

Passo 1: Escolha $a=a_{1}+a_{2} i, b=b_{1}+b_{2} i \in \mathbb{Z}[i]$ e aplique o Algoritmo6.2.

Passo 2: Nas células $B 3$ e C3, digite 1 e 0 , respectivamente.

Passo 3: Nas células $B 4$ e C4, digite 0 e 1, respectivamente.

Passo 4: Na célula D3, digite: =SE(D1=COMPLEXO(0;0);"Acabou";IMSUBTR(B3; IMPROD(C2;C3)))

Passo 5: Na célula D4, digite: =SE(D1=COMPLEXO(0;0);"Acabou";IMSUBTR(B4; IMPROD(C2;C4)))

Passo 6: Arraste a célula D3 sobre as células E3, F3, G3, etc.

Passo 7: Arraste a célula D4 sobre as células E4, F4, G4, etc.

Resultado: Quando em alguma célula da linha 3 (linha 4) aparecer a palavra "Acabou", a célula imediatamente anterior será o valor de $s$ (valor de $t$ ).

Na figura a seguir, vemos como são obtidas as constantes de Bezout para $75+41 i$ e $9+15 i$. Temos que $s=-5-2 i$ e $t=26-4 i$.

Figura $10-$ Constantes de Bezout para $75+41 i$ e $9+15 i$.

\begin{tabular}{|l|c|c|c|c|c|c|c|c|}
\hline 4 & A & B & C & D & E & $F$ & $G$ & $H$ \\
\hline 1 & & $75+41 i$ & $9+15 i$ & $9-i$ & $-2-2 i$ & $1-i$ & 0 & \\
\hline 2 & & $4-2 i$ & $1+2 i$ & $-2+2 i$ & $-2 i$ & Acabou & \\
\hline 3 & & 1 & 0 & 1 & $-1-2 i$ & $-5-2 i$ & Acabou & \\
\hline 4 & & 0 & 1 & $-4+2 i$ & $9+6 i$ & $26-4 i$ & Acabou & \\
\hline 5 & & & & & & & & \\
\hline
\end{tabular}

Fonte: Elaboração dos autores (2020).

\subsection{Soluções de equações diofantinas em $\mathbb{Z}[i]$}

Consideremos uma equação diofantina $a X+b Y=c$ com $a=a_{1}+a_{2} i, b=b_{1}+b_{2} i$ e $c=c_{1}+c_{2} i$ em $\mathbb{Z}[i]$. Para determinarmos uma solução $x_{0}, y_{0} \in \mathbb{Z}[i]$, por meio de uma planilha eletrônica, aplicamos o algoritmo a seguir. 


\section{Algoritmo 6.4.}

Passo 1: Aplique o Algoritmo6.3 para $a$ e $b$.

Passo 2: Na célula B5, digite: $=$ COMPLEXO $\left(c_{1} ; c_{2}\right)$

Passo 3: Na célula C5, digite:

$=S E(D 1=C O M P L E X O(0 ; 0) ; \operatorname{IMPROD}(1 ; C 1) ; S E(E 1=C O M P L E X O(0 ; 0) ; \operatorname{IMPROD}(1 ; D 1)$;

SE(F1=COMPLEXO(0;0);IMPROD(1;E1);SE(G1=COMPLEXO(0;0);IMPROD(1;F1);

$S E(H 1=C O M P L E X O(0 ; 0) ; \operatorname{IMPROD}(1 ; G 1) ; \operatorname{SE}(I 1=\operatorname{COMPLEXO}(0 ; 0) ; \operatorname{IMPROD}(1 ; H 1)$;

SE(J1=COMPLEXO(0;0);IMPROD(1;/1);SE(L1=COMPLEXO(0;0);IMPROD(1;K1);

SE(M1=COMPLEXO(0;0);IMPROD(1;L1);SE(N1=COMPLEXO(0;0);IMPROD(1;M1);

SE(O1=COMPLEXO(0;0);IMPROD(1;N1);SE(P1=COMPLEXO(0;0);IMPROD(1;01);0))))))))))))

Passo 4: Copie a célula C2 e cole-a na célula C6.

Passo 5: Copie a célula D1 e cole-a na célula D5.

Passo 6: Na célula B7, digite:

$$
\begin{aligned}
& =S E(D 3=\text { "Acabou";IMPROD(1;C3);SE(E3="Acabou";IMPROD(1;D3); } \\
& \text { SE(F3="Acabou";IMPROD(1;E3);SE(G3="Acabou";IMPROD(1;F3); } \\
& \text { SE(H3=“Acabou”;IMPROD(1;G3);SE(I3=“Acabou”;IMPROD(1;H3); } \\
& \text { SE(K3="Acabou";IMPROD(1;J3);SE(L3=“Acabou”;IMPROD(1;K3); } \\
& \text { SE(M3="Acabou";IMPROD(1;L3);SE(N3="Acabou”;IMPROD(1;M3); } \\
& \text { SE(O3=“Acabou";(MPROD(1;N3);0))))))))))) }
\end{aligned}
$$

Passo 7: Copie a célula B7 e cole-a na célula B8.

Passo 8: Na célula B9, digite: =SE(D5=COMPLEXO(0;0);IMPROD(C6;B7);“Eq. Sem Sol”)

Passo 9: Na célula B10, digite: =SE(D5=COMPLEXO(0;0);IMPROD(C6;B8);“Eq. Sem Sol”)

Resultado: Se nas células B9 e B10 aparecerem a expressão "Eq Sem Sol.", então a equação $a X+b Y=c$ não tem solução. Caso contrário, os inteiros gaussianos que aparecem serão, respectivamente, soluções iniciais $x_{0}$ e $y_{0}$ da equação.

Observação 6.5. Cabe aqui explicar o que os passos do algoritmo anterior resultam.

- O passo 3 desloca o mdc(a,b) da linha 1 para a célula C5.

- Os passo 4 e 5 resultam, respectivamente, o quociente e o resto da divisão de $c$ por $m d c(a, b)$.

- Os passos 6 e 7 deslocam as constantes de Bezout das linhas 3 e 4 para as células B7 e B8. 
- Os passos 8 e 9 nos dão as soluções iniciais da equação, caso elas existam.

- O algoritmo identifica, no passo 5, se o resto da divisão de $c$ por $m d c(a, b)$ é igual ou diferente de 0. Caso tal resto não seja 0, o algoritmo fornece a resposta "Eq. Sem Sol.", o que significa que a equação dada não tem solução.

Considere as equações $(75+41) X+(9+15 i) Y=7+i$ e $(75+41) X+(9+15 i) Y=7+2 i$. As próximas figuras mostram que a primeira equação tem solução $x_{0}=-7-26 i, y_{0}=94+92 i$ e a segunda equação não tem solução.

Figura $11-(75+41) X+(9+15 i) Y=7+i$ tem solução.

\begin{tabular}{|c|c|c|c|c|c|c|c|c|}
\hline \multicolumn{1}{|c|}{$\mathrm{A}$} & $\mathrm{B}$ & $\mathrm{C}$ & $\mathrm{D}$ & $\mathrm{E}$ & $\mathrm{F}$ & $\mathrm{G}$ & $\mathrm{H}$ \\
\hline 1 & & $75+41 \mathrm{i}$ & $9+15 \mathrm{i}$ & $9-\mathrm{i}$ & $-2-2 i$ & $1-i$ & 0 & \\
\hline 2 & & & $4-2 i$ & $1+2 i$ & $-2+2 i$ & $-2 i$ & Acabou & \\
\hline 3 & & 1 & 0 & 1 & $-1-2 i$ & $-5-2 i$ & Acabou & \\
\hline 4 & & 0 & 1 & $-4+2 i$ & $9+6 i$ & $26-4 i$ & Acabou & \\
\hline 5 & & $7+\mathrm{i}$ & $1-i$ & 0 & & & & \\
\hline 6 & & & $3+4 i$ & & & & & \\
\hline 7 & $\mathrm{~s}$ & $-5-2 i$ & & & & & & \\
\hline 8 & $\mathrm{t}$ & $26-4 i$ & & & & & & \\
\hline 9 & $\mathrm{x}_{0}$ & $-7-26 i$ & & & & & & \\
\hline 10 & $\mathrm{y}_{0}$ & $94+92 i$ & & & & & & \\
\hline 11 & & & & & & & & \\
\hline
\end{tabular}

Fonte: Elaboração dos autores (2020).

Figura $12-(75+41) X+(9+15 i) Y=7+2 i$ não tem solução.

\begin{tabular}{|c|c|c|c|c|c|c|c|c|}
\hline \multicolumn{1}{|c|}{$\mathrm{A}$} & $\mathrm{B}$ & $\mathrm{C}$ & $\mathrm{D}$ & $\mathrm{E}$ & $\mathrm{F}$ & $\mathrm{G}$ & $\mathrm{H}$ \\
\hline 1 & & $75+41 \mathrm{i}$ & $9+15 \mathrm{i}$ & $9-\mathrm{i}$ & $-2-2 \mathrm{i}$ & $1-i$ & 0 & \\
\hline 2 & & & $4-2 \mathrm{i}$ & $1+2 \mathrm{i}$ & $-2+2 \mathrm{i}$ & $-2 \mathrm{i}$ & Acabou & \\
\hline 3 & & 1 & 0 & 1 & $-1-2 \mathrm{i}$ & $-5-2 \mathrm{i}$ & Acabou & \\
\hline 4 & & 0 & 1 & $-4+2 \mathrm{i}$ & $9+6 \mathrm{i}$ & $26-4 \mathrm{i}$ & Acabou & \\
\hline 5 & & $7+2 \mathrm{i}$ & $1-\mathrm{i}$ & 1 & & & & \\
\hline 6 & & & $2+4 \mathrm{i}$ & & & & & \\
\hline 7 & $\mathrm{~s}$ & $-5-2 \mathrm{i}$ & & & & & & \\
\hline 8 & $\mathrm{t}$ & $26-4 \mathrm{i}$ & & & & & & \\
\hline 9 & $\mathrm{x}_{0}$ & Eq. Sem Sol. & & & & & & \\
\hline 10 & $\mathrm{y}_{0}$ & Eq. Sem Sol. & & & & & & \\
\hline 11 & & & & & & & & \\
\hline
\end{tabular}

Fonte: Elaboração dos autores (2020). 
O próximo algoritmo visa determinar outras soluções de $a X+b Y=c$, via uma planilha eletrônica. Estas soluções são determinadas via as fórmulas dadas pelo Teorema $4.3($ iii).

\section{Algoritmo 6.6.}

Passo 1: Aplique o Algoritmo 6.4 para equação $a X+b Y=c$.

Passo 2: Na célula B11, digite:

$=C O M P L E X O(S E(A B S(A R R E D O N D A R . P A R A . B A I X O(I M R E A L(I M D I V(B 1 ; C 5)) ; 0)$

-IMREAL(IMDIV(B1;C5)))>0,5;ARREDONDAR.PARA.CIMA(IMREAL(IMDIV(B1;C5));0);

ARREDONDAR.PARA.BAIXO(IMREAL(IMDIV(B1;C5));0));SE(ABS(ARREDONDAR.PARA.BAIXO

(IMAGINÁRIO(IMDIV(B1;C5));0)-IMAGINÁRIO(IMDIV(B1;C5)))>0,5;ARREDONDAR.PARA.CIMA

(IMAGINÁRIO(IMDIV(B1;C5));0);ARREDONDAR.PARA.BAIXO(IMAGINÁRIO(IMDIV(B1;C5));0)))

Passo 3: Na célula B12, digite:

$=$ COMPLEXO(SE(ABS(ARREDONDAR.PARA.BAIXO(IMREAL(IMDIV(C1;C5));0)

-IMREAL(IMDIV(C1;C5)))>0,5;ARREDONDAR.PARA.CIMA(IMREAL(IMDIV(C1;C5));0);

ARREDONDAR.PARA.BAIXO(IMREAL(IMDIV(C1;C5));0));SE(ABS(ARREDONDAR.PARA.BAIXO (IMAGINÁRIO(IMDIV(C1;C5));0)-IMAGINÁRIO(IMDIV(C1;C5)))>0,5;ARREDONDAR.PARA.CIMA (IMAGINÁRIO(IMDIV(C1;C5));0);ARREDONDAR.PARA.BAIXO(IMAGINÁRIO(IMDIV(C1;C5));0)))

Passo 4: Escolha um inteiro gaussiano $z=z_{1}+z_{2} i$ e, na célula $B 13$, digite: $=\operatorname{COMPLEXO}\left(z_{1} ; z_{2}\right)$

Passo 5: Na célula B14, digite: =IMSOMA(B9;IMPROD(B12;B13))

Passo 6: Na célula B15, digite: =IMSUBTR(B10;IMPROD(B11;B13))

Resultado: As células B14 e B15 retornam valores de $x$ e y que são soluções de $a X+b Y=c$.

Observação 6.7. No Algoritmo 6.6. os passos 2 e 3 calculam $\frac{a}{d}$ e $\frac{b}{d}$, respectivamente. Os passos 5 e 6 determinam, respectivamente, soluções $x=x_{0}+\frac{b}{d} z$ e $y=y_{0}-\frac{a}{d} z$ para cada $z \in \mathbb{Z}[i]$ que for digitado na célula $B 13$.

Exemplo 6.8. A próxima figura mostra todos os algoritmos descritos nesta seção serem aplicados para a equação $(75+41 i) X+(9+15 i) Y=7+i$. Temos que $m d c(75+41 i, 9+15 i)=1-i$ e $1-i \mid 7+i$. Também, $x_{0}=-7-26 i, y_{0}=94+92 i, \frac{a}{d}=17+58 i e \frac{b}{d}=-3+12 i$. Por fim, $x=-28+7 i$ e $y=101-99 i$ são soluções obtidas a partir de $z=3+i$. 
Figura 13-Outra solução da equação $(75+41) X+(9+15 i) Y=7+i$

\begin{tabular}{|c|c|c|c|c|c|c|c|}
\hline & $\mathrm{A}$ & $\mathrm{B}$ & $\mathrm{C}$ & $\mathrm{D}$ & $\mathrm{E}$ & $\mathrm{F}$ & $\mathrm{G}$ \\
\hline 1 & & $75+41 \mathrm{i}$ & $9+15 \mathrm{i}$ & $9-\mathrm{i}$ & $-2-2 i$ & $1-i$ & 0 \\
\hline 2 & & & $4-2 i$ & $1+2 i$ & $-2+2 i$ & $-2 i$ & Acabou \\
\hline 3 & & 1 & 0 & 1 & $-1-2 i$ & $-5-2 i$ & Acabou \\
\hline 4 & & 0 & 1 & $-4+2 i$ & $9+6 i$ & $26-4 i$ & Acabou \\
\hline 5 & & $7+i$ & $1-i$ & 0 & & & \\
\hline 6 & & & $3+4 i$ & & & & \\
\hline 7 & $\mathrm{~s}$ & $-5-2 i$ & & & & & \\
\hline 8 & $\mathrm{t}$ & $26-4 i$ & & & & & \\
\hline 9 & $\mathrm{x}_{0}$ & $-7-26 i$ & & & & & \\
\hline 10 & $y_{0}$ & $94+92 i$ & & & & & \\
\hline 11 & $\mathrm{a} / \mathrm{d}$ & $17+58 \mathrm{i}$ & & & & & \\
\hline 12 & $\mathrm{~b} / \mathrm{d}$ & $-3+12 i$ & & & & & \\
\hline 13 & $\mathrm{z}$ & $3+i$ & & & & & \\
\hline 14 & $\mathrm{x}$ & $-28+7 i$ & & & & & \\
\hline 15 & $\mathrm{y}$ & $101-99 i$ & & & & & \\
\hline 16 & & & & & & & \\
\hline
\end{tabular}

Fonte: Elaboração dos autores (2020).

\section{Conclusão}

Determinar um $m d c$ entre dois elementos de um domínio qualquer pode ser trabalhoso. Isso se deve ao fato da definição de $m d c$ não apresentar um possível candidato. Ela apenas diz que $d$ é um $m d c$ de $a$ e $b$, se $d$ é um divisor comum de $a$ e $b$ e qualquer outro divisor comum de $a$ e $b$ divide $d$. Pela Observação 3.7, quando estamos trabalhando com domínios euclidianos, isso fica um pouco mais fácil, porém, podemos ter dificuldades nos cálculos das sucessivas divisões. Para determinarmos as constantes de Bezout e as soluções de equações diofantinas, também podemos encontrar dificuldades nos cálculos. Por isso, um programa de computador que realize tais cálculos é crucial.

Nesse sentido, os algoritmos para $\mathbb{Z}[i]$, que apresentamos na Seção 6, são interessantes e muito práticos, pois eles podem ser aplicados praticamente em qualquer computador, smartphone ou tablet sem a necessidade do conhecimento de uma linguagem de programação específica. Basta ter instalado o Microsoft Office ou o LibreOffice e seguir os passos apresentados nos algoritmos da Seção 6. Observamos que uma vez programada uma tabela conforme descrevemos anteriormente, faz-se necessário, somente, alterar as células B1, C1, B5 e B13 para determinar diferentes $m d c^{\prime} s$ ou resolver diferentes equações diofantinas. 
Durante o desenvolvimento deste trabalho, percebemos a falta de recursos computacionais simples, viáveis e assecíveis para a realização dos cálculos algébricos em $\mathbb{Z}[i]$ que necessitávamos. Com essa necessidade passamos considerar as planilhas do LibreOffice como uma possibilidade de ferramenta. Nós as escolhemos por vários motivos, dentre eles estão a facilidade de acesso e manuseio pelos usuários, bem como a existência de algumas funções pré-definidas, por exemplo, multiplicação, adição e subtração de números complexos. Por um lado, para nós, o mais importante foi a programação da divisão euclidiana em $\mathbb{Z}[i]$. Por outro lado, como pode-se perceber no Passo 4 do Algoritmo 6.1, esta programação foi a mais difícil, pois envolve diversos comandos lógicos.

Nós acreditamos que um aplicativo de celular como aquele desenvolvido em Santos (2016) seria muito mais atrativo. Porém, ele se limita a smartphones com sistema Android. Ressaltamos ainda que as planilhas que apresentamos também podem ser usadas para resolver equações diofantinas clássicas, ou seja, com coeficientes em $\mathbb{Z}$. Para tanto, basta zerar a parte imaginária dos coeficientes e termo independente da equação.

Esperamos que estudantes e professores de graduação e pós-graduação se inspirem em utilizar as planilhas do LibreOffice para outras atividades. Por exemplo, em Jesus (2018) o autor descreve como tais planilhas podem ser úteis para o estudo de Geometria Analítica. Elas são viáveis para resolver sistemas lineares com poucas incógnitas por meio da Regra de Cramer, pois essa regra depende apenas de cálculos de determinantes e quociente de números reais, que podem ser realizados facilmente em uma planilha eletrônica. Além disso, acreditamos que equações diofantinas sobre outros domínios euclidianos do tipo $\mathbb{Z}[\alpha]$, como descritos no Exemplo 2.2, possam ser estudados com o auxílio de tabelas do LibreOffice.

\section{Referências}

ANDERSON, M.; FEIL, T. A First Course in Abstract Algebra: Rings, Groups and Fields. 3. ed. New York: CRC Press, 2015.

GATHEN, J. V. Z.; GERHARD, J. Modern Computer Algebra. 3. ed. New York: Cambridge University Press, New York, 2013.

HEFEZ, A. Aritmética. 2. ed. Rio de Janeiro: SBM-Coleção PROFMAT, 2016.

JESUS, O. F. de. O Uso de Planilhas do Excel Aplicadas a Tópicos de Geometria Analítica. 2018. 243f. Dissertação (Mestrado em Matemática) - Programa de Mestrado Profissional em Matemática em rede Nacional, Universidade Federal de Goias, Regional de Jataí, Jataí, 2018. 
LIBREOFFICE. Funções de suplemento (add-in), lista das funções de análise - parte 2. LibreOffice Help. Disponível em: https://help.libreoffice.org/3.3/Calc/Add-in_Functions, _List_of_Analysis_Functions_Part_Two/pt-BR. Acesso em: 17 mar. 2020.

MILIES, C. F. P. M.; COELHO, S. P. Números: Uma Introdução à Matemática. 3. ed. São Paulo: USP, 2001.

SANTOS, L. A. dos. Equações diofantinas lineares: um aplicativo para a resolução. 2016. 65f. Trabalho de Conclusão de Curso (Licenciatura em Matemática) - Universidade Estadual Paulista, Guaratinguetá, 2016.

SEMATIC SCHOLAR. Solution of simple diophantine equations by means of MATLAB. Disponível em: https: //www. semanticscholar.org/paper/SOLUTION-OF-SIMPLE-DIOPHANTINE-EQUATIONS-BY-MEANS/ 6ec1d1d98bb1044fb0683540474b3dadca04bfe0. Acesso em: 25 set. 2019. 